\title{
Painless Thyroiditis Induced by Immune Checkpoint Inhibitors in a Patient with Small Cell Lung Cancer
}

\section{Maria Jose Burchés Feliciano*, Antonia Perez Lazaro and Juan Francisco Merino Torres}

Servicio de Endocrinología y Nutrición, Hospital Universitari i Politècnic La Fe Valencia, Spain

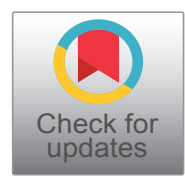

*Corresponding author: Maria Jose Burches Feliciano, Servicio de Endocrinología y Nutrición, Hospital Universitari i Politècnic La Fe Valencia, Spain

New antineoplastic agents consisting of specific monoclonal antibodies which enhance the host's immune response against the tumoral cells are becoming more common in clinical practice. Ipilimumab, an antibody that blocks the cytotoxic T-lymphocyte antigen 4 (CTLA-4), and nivolumab, a programmed cell death 1 (PD-1) inhibitor are now approved for several advanced neoplasias such as melanoma, non-small cell lung cancer and renal cell carcinoma [1]. Likewise, ongoing clinical trials are being developed to extend their use to other malignancies. However, due to the mechanism of action of these drugs, several autoimmune adverse effects may occur [2]. Clinicians should be aware of immunotherapy-related adverse events as their use is expected to increase exponentially in the near future. In this paper we present a case of checkpoint inhibitor-induced thyroiditis in a patient with metastatic small cell lung cancer (SCLC).

A 64-year-old male was diagnosed with extensive-stage small cell lung cancer and treated with six cycles of cisplatin and etoposide followed by whole brain and thorax radiation therapy. Post-treatment evaluation revealed disease progression hence he was enrolled in a clinical trial that combined immunotherapy with ipilimumab and nivolumab (every three weeks for four cycles). Thyroid function tests performed before the first dose were normal [thyroid stimulating hormone (TSH) $0.978 \mathrm{mU} / \mathrm{L}$ (0.350-4.940), free thyroxine (FT4) $0.87 \mathrm{ng} / \mathrm{dl}(0.70-1.48)]$ and a new screening before the second cycle did not reveal major changes.

However, two weeks after the second dose the pa-
Figure 1: Thyroid scan demonstrated a low uptake of the radiotracer.

tient reported progressive weakness, fatigue, palpitations and significant weight loss. He denied diarrhea, heat intolerance or decreased oral intake. There was no personal or family history of thyroid disease. Medications known to interfere with thyroid function were ruled out. Blood pressure was $124 / 67 \mathrm{mmHg}$, heart rate within the normal range at $72 \mathrm{bpm}$, his body temperature was $35.8^{\circ} \mathrm{C}$ and no proptosis or hand tremor was observed. There was no enlargement or tenderness of the thyroid gland on exam. Blood tests demonstrated a suppressed TSH at $0.0012 \mathrm{mU} / \mathrm{L}$ along with raised $\mathrm{FT} 4$ at $3.6 \mathrm{ng} / \mathrm{dl}$ and free tri-iodothyronine (FT3) of $9.91 \mathrm{pg} / \mathrm{dl}$ (1.70-3.70). Thyroid peroxidase (TPO) and thyroid stimulating immunoglobulin (TSI) antibodies were found to

Citation: Burches-Feliciano MJ, Pérez-Lázaro A, Merino-Torres JF (2018) Painless Thyroiditis Induced by Immune Checkpoint Inhibitors in a Patient with Small Cell Lung Cancer. Clin Med Rev Case Rep 5:235. doi.org/10.23937/2378-3656/1410235

Accepted: September 12, 2018: Published: September 14, 2018

Copyright: (c) 2018 Burches-Feliciano MJ, et al. This is an open-access article distributed under the terms of the Creative Commons Attribution License, which permits unrestricted use, distribution, and reproduction in any medium, provided the original author and source are credited. 
Table 1: TSH and T4L levels during nivolumab and ipilimumab treatment.

\begin{tabular}{|l|l|l|l|l|l|}
\hline & Week 0 & Week 3 & Week 5 & Week 8 & Week 11 \\
\hline TSH (mU/L) & 0.978 & 0.982 & 0.0012 & 0.144 & 0.846 \\
\hline T4L (ng/dl) & 0.87 & 0.89 & 3.60 & 0.77 & 0.82 \\
\hline
\end{tabular}

be low at $0.94 \mathrm{U} / \mathrm{mL}(0.05-6.00)$ and $0.50 \mathrm{UI} / \mathrm{L}(0.00-$ 1.99), respectively. A thyroid scan revealed decreased uptake of the radiotracer, consistent with thyroiditis (Figure 1).

He was diagnosed with painless thyroiditis induced by ipilimumab and nivolumab and started on methimazole $15 \mathrm{mg}$ per day. Weekly evaluation confirmed gradual improvement of the symptoms. Normalization of FT4 levels occurred three weeks later and anti-thyroid drugs (ATDs) were discontinued. Six weeks after initial evaluation full biochemical resolution of hyperthyroidism was achieved. Suspension of immunotherapy was not needed. The short length of the disease suggests that resolution might not have been related to the use of ATDs (Table 1).

Checkpoint blocking antibodies targeting PD-1 receptor and CTLA-4 have become a promising therapeutic option for other metastatic tumors besides melanoma. The combination of nivolumab plus ipilimumab has shown antitumor activity in patients with metastatic SCLC who have progressed after first-line treatment [3] and their effectiveness is currently being tested in phase 3 clinical trials.

Under physiological conditions, immune checkpoints help to maintain self-tolerance, prevent autoimmunity and protect tissues from damage during the inflammatory response to infection. New immunomodulatory anticancer drugs induce T-cell activation and proliferation resulting in significant endogenous antitumor activity, yet this immunological boosting might also cause a wide range of immune-mediated adverse effects. These socalled immune-related adverse events (irAEs) are typically transient and can theoretically involve any organ system, being the most commonly affected the skin and the gastrointestinal tract [4].

Endocrine toxicity is thought to occur in less than $20 \%$ of patients, although recent studies have shown a higher incidence in those on combination therapy (up to $25 \%$ ) compared to monotherapy [5]. Diagnosis can be challenging due to the unspecific character of the symptoms (fatigue, anorexia, nausea or headache), which may overlap with those related to the malignant tumor. Reported endocrinopathies include hypophysitis, thyroid disorders, adrenal insufficiency and rarely, type 1 diabetes mellitus.

Thyroid irAEs are often asymptomatic and diagnosis is made incidentally by routine laboratory tests. The mean time to presentation is two to four months from drug commencement, however, the combination therapy has been associated with an earlier onset. Despite the association between thyroid dysfunction and the total number of doses that some studies have suggested, risk factors are still unknown [6]. Overall, thyroid side effects induced by checkpoint inhibitors are seen in nearly $15 \%$ of patients but the incidence rises in those treated with the combination regimen, affecting one out of five.

Patients can present with both hypo- and hyperthyroidism. Hypothyroidism has been more frequently associated with PD-1 inhibitors. Among the causes of hormone excess, both Graves' disease and thyroiditis have been described. Painless thyroiditis is characterized by transient thyrotoxicosis frequently followed by a hypothyroid phase that might be permanent [7]. Graves' disease is more likely to occur on ipilimumab therapy, although the number of reported cases appears to be small [8].

Clinical guidelines recommend thyroid function assessment at baseline and prior to each dose. Abnormal results should be followed by an accurate history, physical examination and further evaluation to identify the etiology. If hyperthyroidism is observed, measurement of autoantibodies as well as a radioiodine or technetium imaging study might be useful in the diagnostic approach. A low uptake of the radiotracer along with negative TSI antibodies will be consistent with thyroiditis. TPO and anti-thyroglobulin antibodies can be present or absent. If thyroid scan is contraindicated or unavailable, ultrasound showing a non-enlarged hypoechogenic gland with normal blood flow can help confirming the diagnosis.

Multidisciplinary management should be enhanced for optimal patient care and referral to an endocrinologist is recommended [9]. Most patients affected by checkpoint inhibitor-induced thyroiditis can be managed expectantly but those who are symptomatic or show frank biochemical abnormalities might need betablockers, ATDs or steroids during the hyperthyroid phase. No relation has been found between the use of medical therapy and the median time to resolution of the disease. Severe or life-threatening thyrotoxicosis may require hospitalization and discontinuation of immunotherapy. Hormone replacement therapy is usually needed if symptomatic hypothyroidism occurs following the hyperthyroid phase [10].

In conclusion, thyroid irAEs are an increasingly common condition associated with the use of new immunoregulatory anticancer drugs, especially when administered in combination. Since appropriate management may prevent from immunotherapy discontinuation, frequent monitoring to achieve an early diagnosis is essential for a better outcome.

\section{Funding Statement}

This research did not receive any specific grant from any funding agency in the public, commercial or not-forprofit sector. 


\section{Declaration of Interest}

The authors declare that there is no conflict of interest that could be perceived as prejudicing the impartiality of the research reported.

\section{References}

1. Larkin J, Chiarion-Sileni V, Gonzalez R, Grob JJ, Cowey CL, et al. (2015) Combined Nivolumab and Ipilimumab or monotherapy in untreated melanoma. N Engl J Med 373: 23-34.

2. Corsello SM, Barnabei A, Marchetti P, De Vecchis L, Salvatori $\mathrm{R}$, et al. (2013) Endocrine side effects induced by immune checkpoint inhibitors. J Clin Endocrinol Metab 98: 1361-1375.

3. Antonia SJ, López-Martin JA, Bendell J, Ott PA, Taylor M, et al. (2016) Nivolumab alone and nivolumab plus ipilimumab in recurrent small-cell lung cancer (CheckMate 032): Amulticentre, open-label, phase 1/2 trial. Lancet Oncol 17: 883-895.

4. Ryder M, Callahan M, Postow MA, Wolchok J, Fagin JA (2014) Endocrine-related adverse events following ipilimumab in patients with advanced melanoma: A comprehensive retrospective review from a single institution. Endocr Relat Cancer 21: 371-381.
5. Scott E, Long GV, Guminski A, Clifton-Bligh RJ, Menzies AM, et al. (2018) The spectrum, incidence, kinetics, and management of endocrinopathies with immune checkpoint inhibitors for metastatic melanoma. Eur J Endocrinol 178: 175-182.

6. Yamazaki H, Iwasaki H, Yamashita T, Yoshida T, Suganuma N, et al. (2017) Potential risk factors for Nivolumab-induced thyroid dysfunction. In Vivo 31: 1225-1228.

7. Guaraldi F, Selva RL, Samà MT, D'Angelo V, Gori D, et al. (2017) Characterization and implications of thyroid dysfunction induced by immune checkpoint inhibitors in real-life clinical practice: A long-term prospective study from a referral institution. J Endocrinol Invest 41: 549-556.

8. Azmat U, Liebner D, Joehlin-Price A, Agrawal A, Nabhan F (2016) Treatment of Ipilimumab Induced Graves' Disease in a Patient with Metastatic Melanoma. Case Rep Endocrinol 2016: 2087525.

9. Gonzalez-Rodriguez E, Rodriguez-Abreu D (2016) Immune checkpoint inhibitors: Review and management of endocrine adverse events. Oncologist 21: 804-816.

10. Yamauchi I, Sakane Y, Fukuda Y, Fujii T, Taura D, et al. (2017) Clinical features of Nivolumab-induced thyroiditis: A case series study. Thyroid 27: 894-901. 